\title{
GLM for Image Steganography Technique
}

\author{
Ahmed S. Nori \\ ahmed.s.nori@uomosul.edu.iq \\ Noor N. Ahmed Thana Gh. Ahmed \\ College of Computer Science and Mathematics \\ University of Mosul, Mosul, Iraq
}

Received on: 15/10/2012

Accepted on: 30/01/2013

\section{ABSTRACT}

An improvement to a Steganography method for hiding data using Gray Level Images is proposed in this paper. The method is an improvement over earlier method named Least Significant Bit (LSB). This method uses the 5th, and 6th bits of pixel value for insertion and retrieval of message by using the same bits of pixel value.

The idea here, deals with images in different sizes and extensions (BMP, JPG, PNG) for obtaining better results and more efficient than its original one (LSB). The selection of pixel locations is done by using the pseudo random number generator, Which is uses the same key for insertion as well as retrieval of process.

The experiments show the Superiority of the new idea through using the performance measures (PSNR, BER, NC).

Keywords: GLM, LSB, BMP, JPG, PNG.

GLM

ثناء غانم أحمد

$$
\text { أحمد سامي نوري }
$$

\section{الملخص}

يعرض هذا البحثث تقنيـة محسنة لتطبيق الإخفاء (إخفـاء المعلومـات) في صـور المستوى الرمـادي.

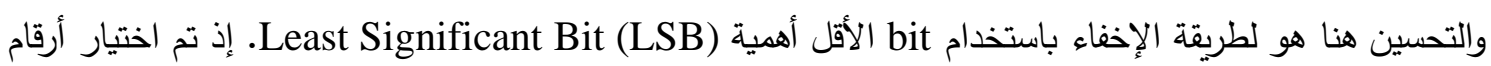

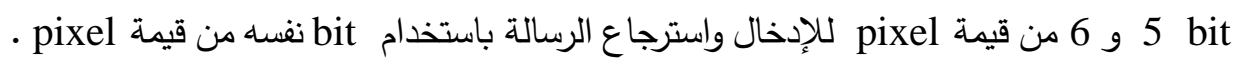

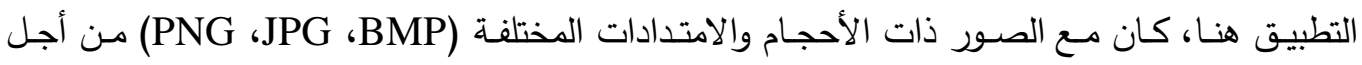
الحصول على نتائج أفضل وأكثر كفاءة من تقنية (LSB) الأصلية. أمسا عملية اختيار المواقع للإخفاء (مواقع

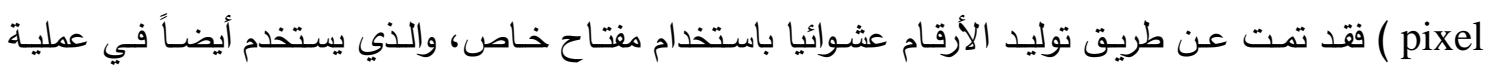

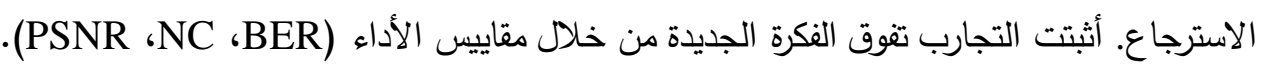

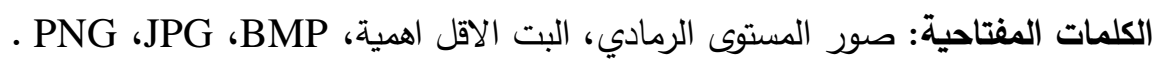

يخلط كثيراً من المبتدئين في العلم المختص بحماية وأمن المعلومات بين علم التشفير وعلم إخفاء المعلومات، معتقدين أن كلا المصطلحين يعطي المعنى نفسه، في حين أن كل مصطلح منهما يغطي علماً خاصاً 
من علوم أمن المعلومات. تهدف تقنية إخفاء المعلومات إلى الإخفاء التام لوجود هذه المعلومات عن طريق إخفائها داخل معلومات أخرى ليست بتلك الأهمية مع الحرص التام على عدم تأثر المعلومات المستخدمة للإخفاء بحقيقة لإنة

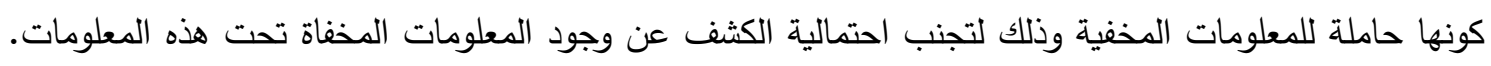
باستخدام هذه الطريقة تقل نسبة الكثف عن المعلومات والعبث بها بنسبة كبيرة؛ لأنه إن كان المهاجم لا يعلم

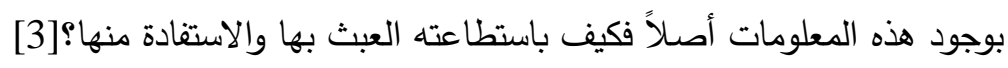

2. 2. علم الإخفاء

يأتي أصل مصطلح علم إخفاء المعلومات (Steganography) من الكلمتين الإغريقيتين: Stegos والتي تعني السقف أو الغطاء و و graphia والتي تعني الكتابة. ويعرف علم إخفاء المعلومات على أنه إخفاء رسالة ما (بيانات) داخل رسالة أخرى (بيانات أخرى) بهدف إخفاء وجود الرسالة الأولى لهدف محدد. والبيانات المستخدمة الخفاء

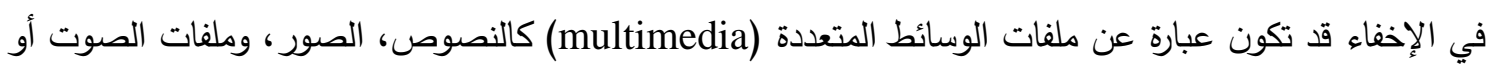
الفيديو وغيرها. وقد تكون أيضا عبارة عن ملفات تنفيذية للبرامج (executable file). وفي عملية الإخفاء نحتاج إلى توفر عنصرين مهمين لإتمام هذه العملية، الأول هو الرسالة التي نهدف إلى إخفائها والثاني هو الغئل الغطاء

[4] المستخدم لإخفاء هذه الرسالة. (cover) 3. المبدأ العام لعلم الإخفاء

أغلب طرائق علم الإخفاء تتبع المبدأ العام التالي: الأشكال (1) و(2) 1- تحليل عناصر ملف الغطاء، وتحضيرها لاستقبال البيانات السرية.

2- تحليل عناصر الملف المطلوب تضمينه (إخفاء بياناته). 3- تطبيق الخوارزمية المناسبة للإخفاء.

4- إرسال ملف الغطاء المتضمن للبيانات السرية من قبل المرسل.

5- استلام ملف الغطاء من قبل الطرف المقصود الإرسال إليه. 6- تحليل عناصر ملف الغطاء واستخراج عناصر الملف السري وفق نفس الخوارزمية المتّبعة في الإخفاء. 7- تجميع البيانات للحصول على الملف السري كاملاً.

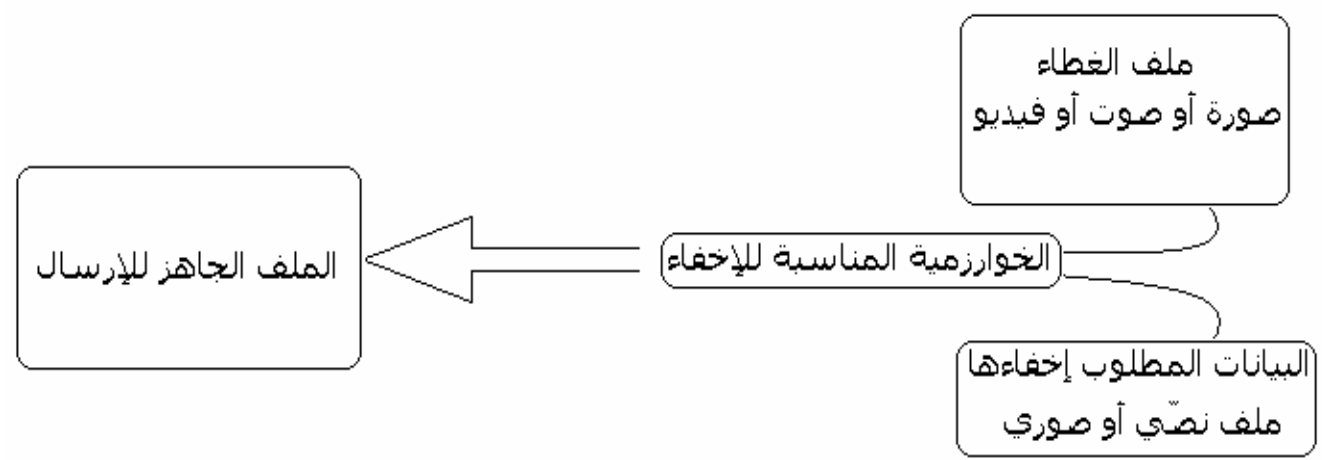

الشكل (1). عملية إخفاء البيانات داخل ملف الغطاء

أن علم التشفير يمكن أن يزيد من قوة العمل، إذ سيصبح من الصعب تحديد وجود إخفاء في الملف الكُرسل،

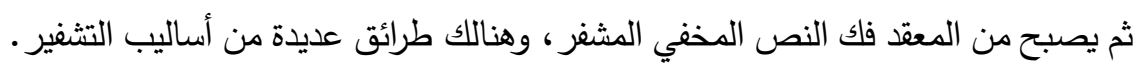




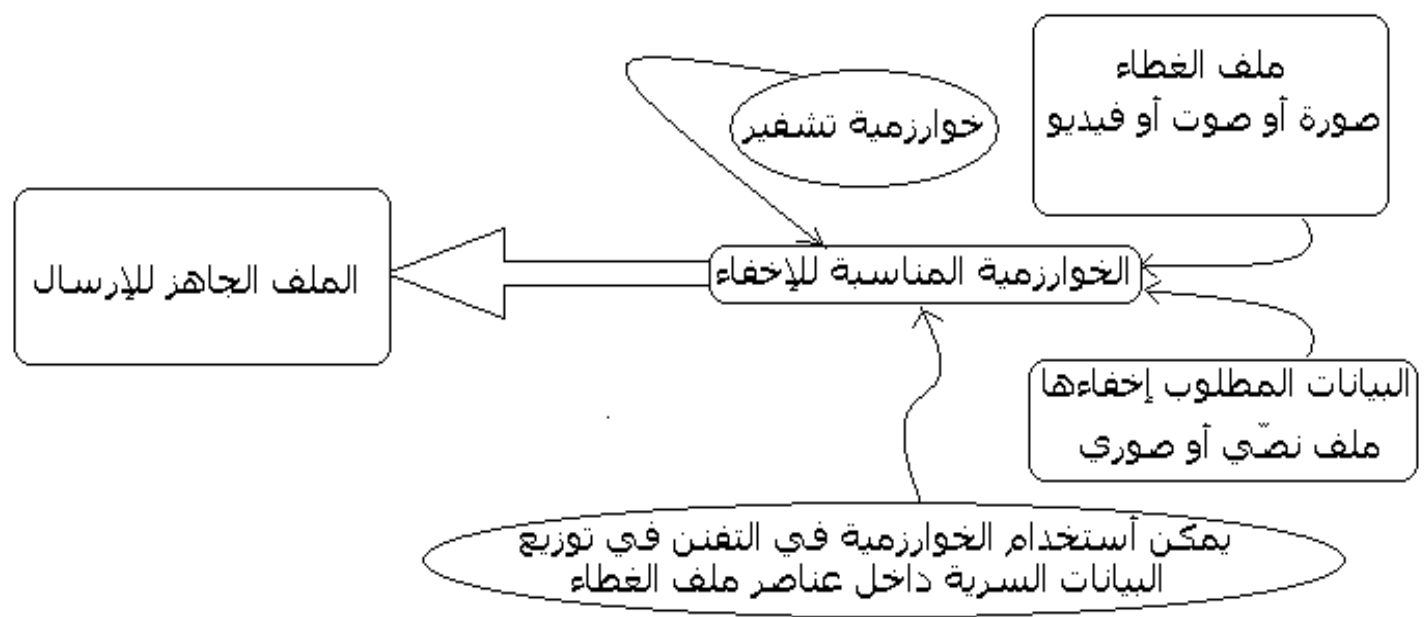

الثكل (2). عملية إخفاء البيانات المشفرة داخل ملف الغطاء

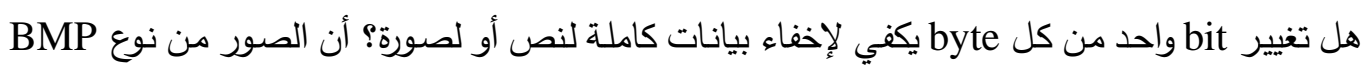

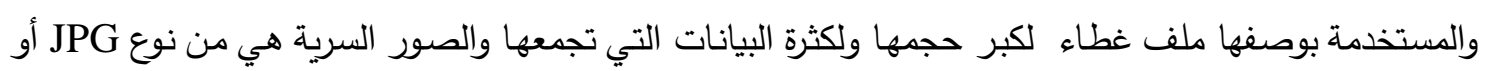

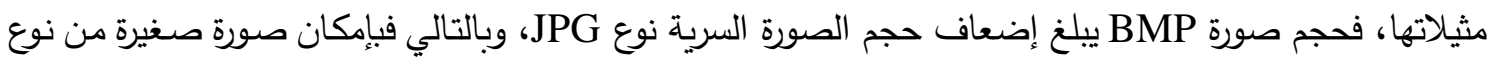
BMP

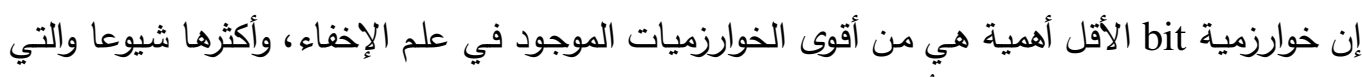

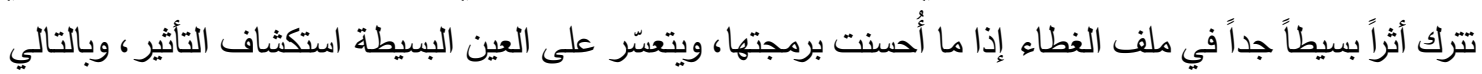

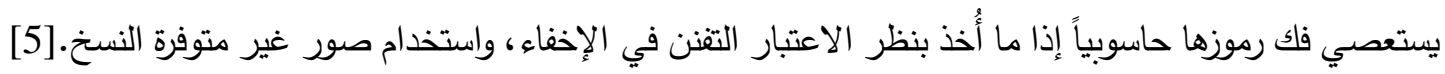
ومن الملاحظ في هذه الطريقة:

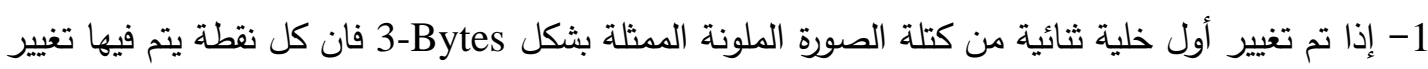
3-Bit 2- عند استخدام الصورة الممثلة 8-Bit فيفضل استخدام الصورة الرمادية، لان التغيير سيكون غير ملحوظاً. في العام (2009) قدمت كل من (فرح باسل احمد وآخرين) فكرة إخفاء باعتماد حماية الملكية في الصور وكانت النتائج جيدة في حينها. [1] أما كل من (رؤى سامي إسماعيل وآخرون) فقد قدموا في العام (2009) بحثاً عن "تطبيقات العلامة المائية

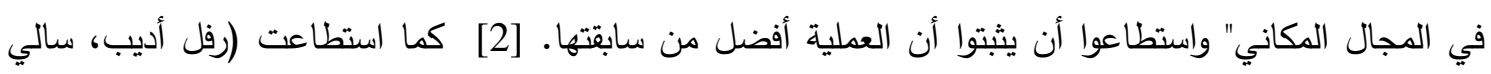

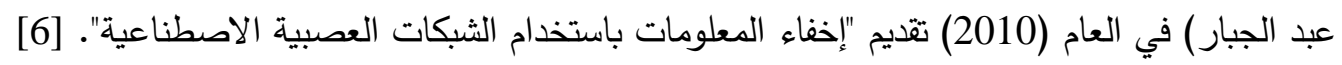
وكذلك Ravi Saini, Rajkumar Yadav) سنة (2011) قدما طريقة مقترحة من خلال "تقنية جديدة

$$
\text { للإخفاء في الصور باستخدام الصور الرمادية". [7] }
$$




\section{5. وصف الطريقة المقترحة}

في الخوارزمية المقترحة سوف نستخدم bit $\left(5^{\text {th }}, 6^{\text {th }}\right.$ من قيم pixel وكما يلي: إذا كان الإدخال (قيمة bit السرية) = صفراً

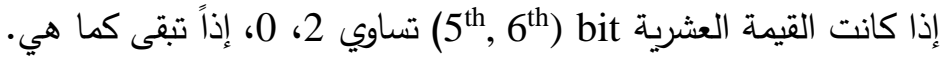

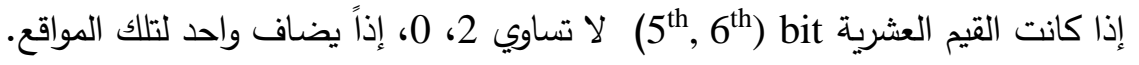
إذا كان الإدخال (قيمة bit السرية) = واحداً

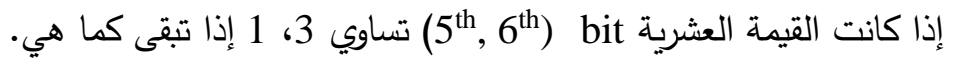
إذا كانت القيمة العشرية bit

$$
\begin{aligned}
& \text { لاسترجاع الرسالة نفحص القيمة العشرية bit }
\end{aligned}
$$

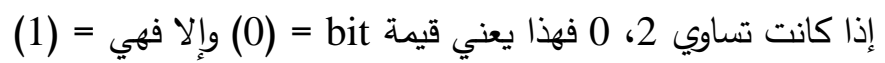

في هذه الخوارزمية سوف نستخدم $\left(5^{\text {th }}, 6^{\text {th }}\right)$ bit لإدخال واسترجاع الرسالة وذلك لإلغاء المساوئ الموجودة في خوارزمية (LSB) والحصول على أفضل النتائج. الأشكال (3) و(4) توضح مخطط عملية الإخفاء والاسترجاع على التوالي. 


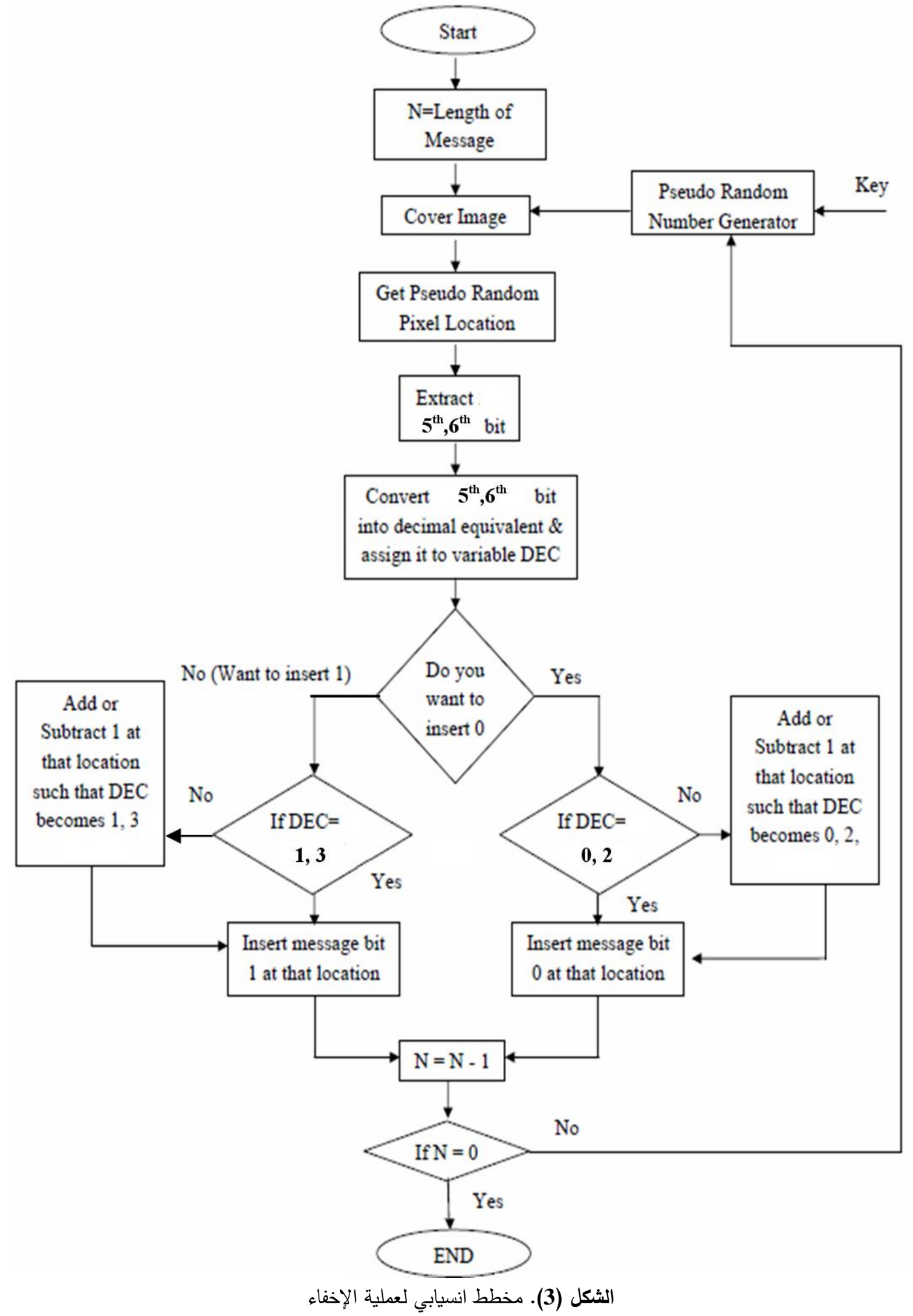




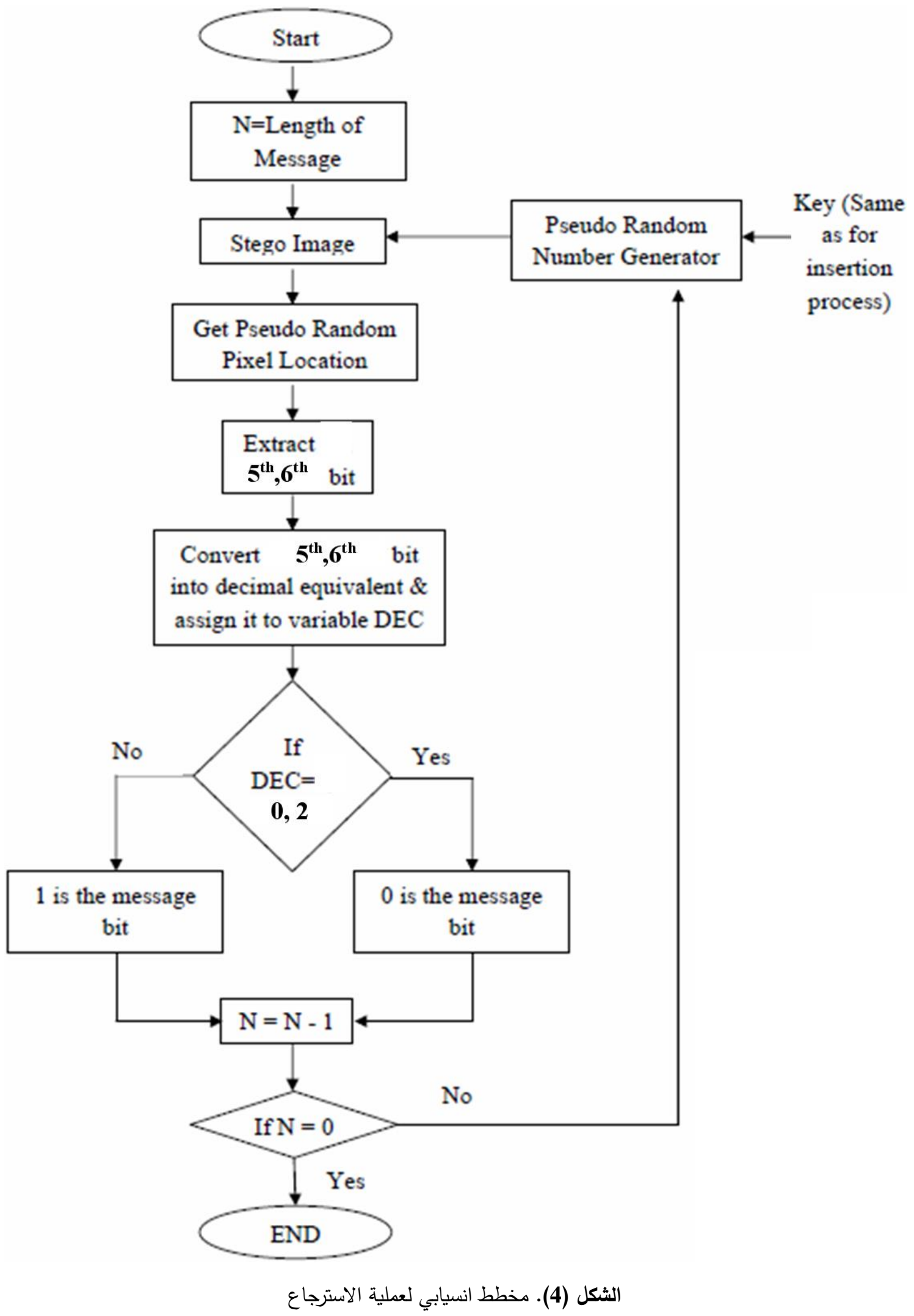


6. مثال عن الخوارزمية المقترحة

تم اخذ صورة وتحويلها إلى التدرج الرمادي ولنفرض أنها تعطي مجموعة pixel الموضحة بالثكل (5) ولنفرض انه مطلوب إخفاء الرسالة 100101 في هذه الصورة فيتم تحديد مجموعة من المواقع العشوائية الأشكال (6، 7) والتي تمثل المفتاح الذي سيستخدم في الإخفاء والاسترجاع.

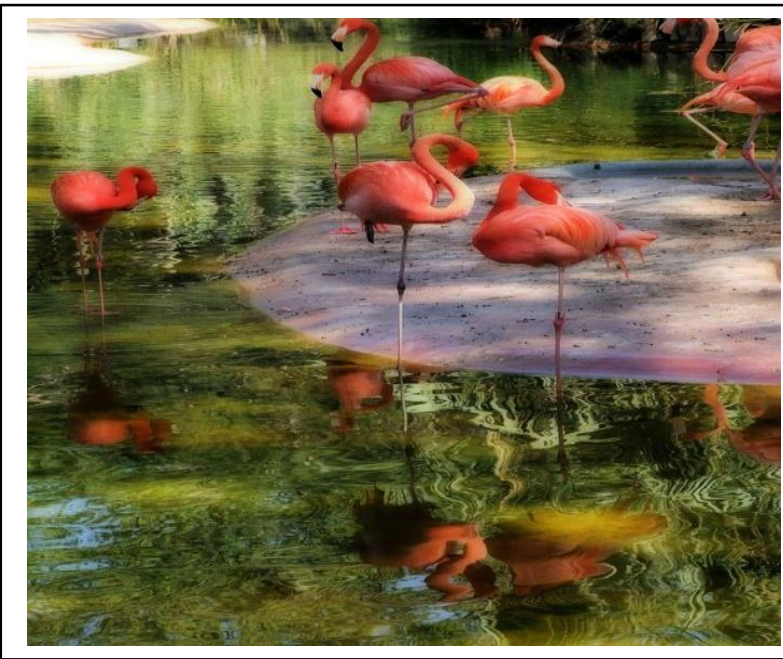

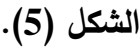

نموذج لتطبيق الخوارزمية

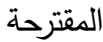

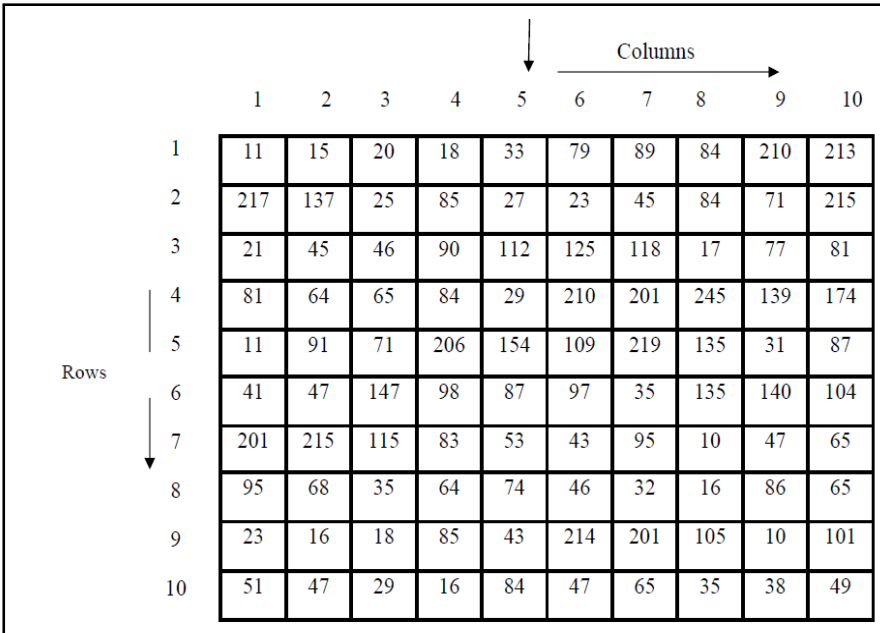

الثكل (6). قيم الترددات الرمادية للصورة المقترحة

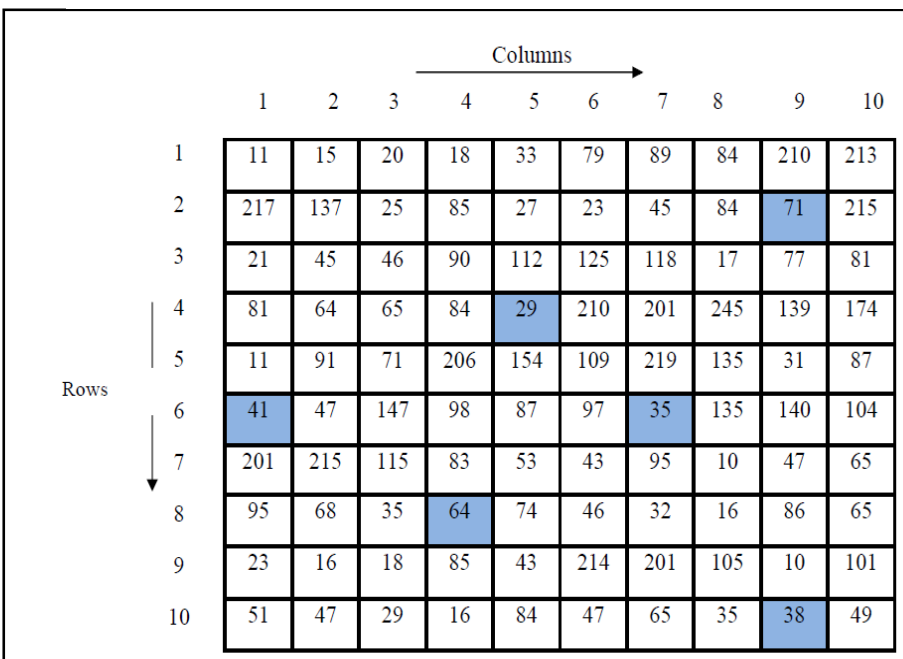

الثكل (7). تحديد المواقع المختارة باستخدام توليد أرقام عشوائية 
L (4, 5), L (6, 1), L (10, 9), L (6, 7), L (2, 9), L (8, 4) والتي تمثل الصف والعمود للمواقع وتم الحصول على القيم: 29, 41, 38, 35, 71 and 64.

يمكن توضيح عملية الحشر بالثكل (8).

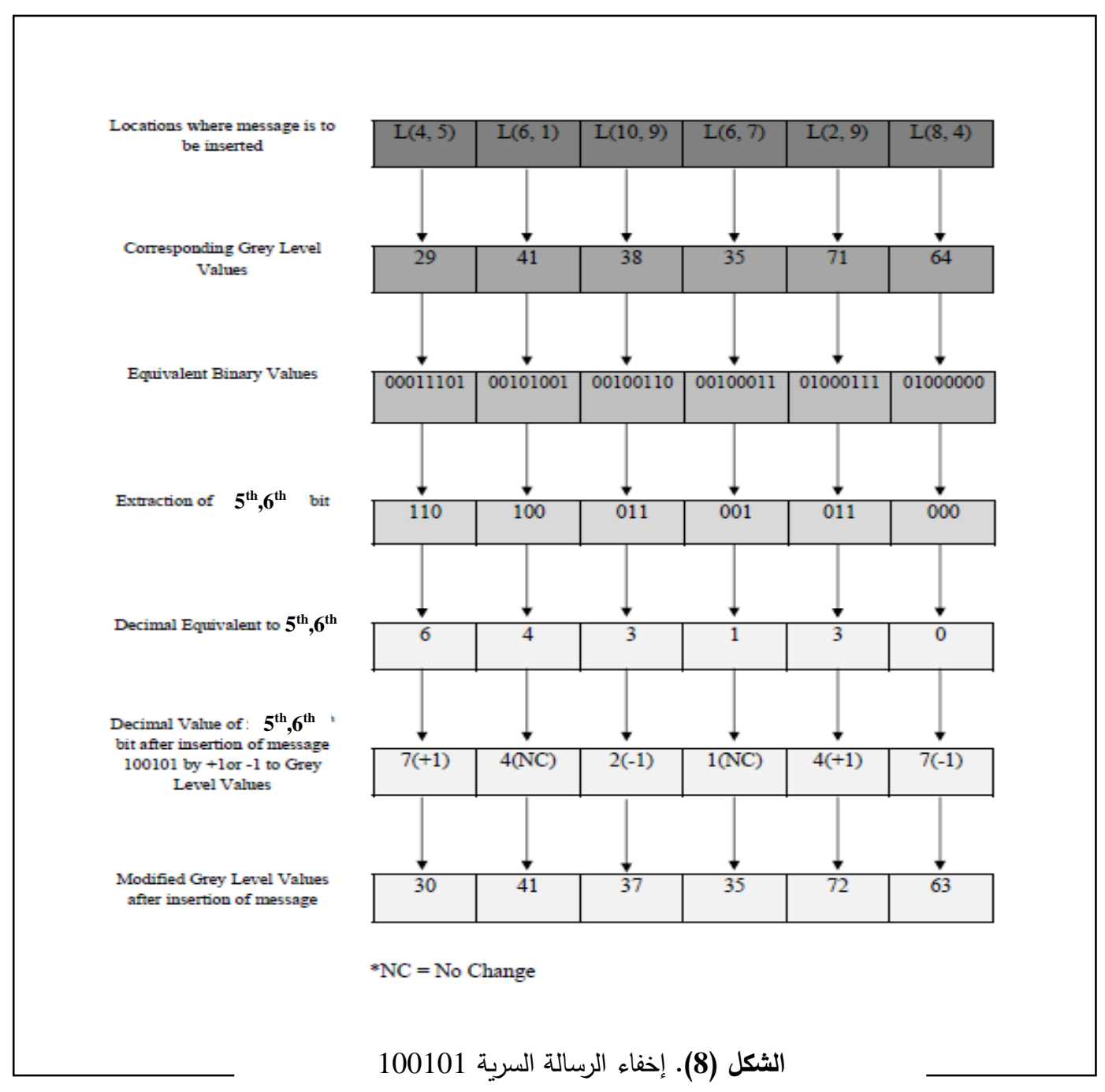

أما عملية الاسترجاع فيمكن توضيحها بالثكل (9). 


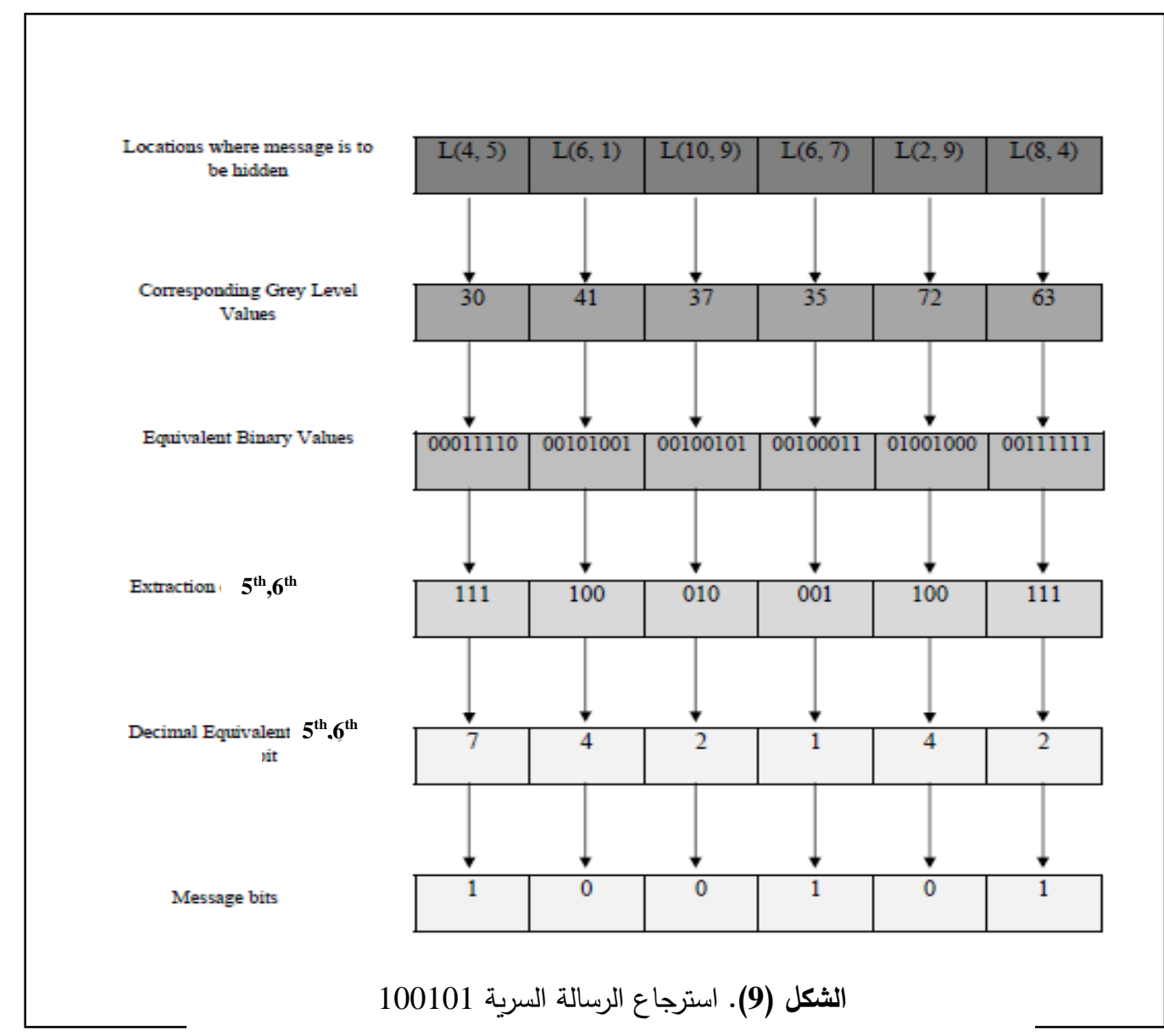

$$
\begin{aligned}
& P S N R=10 \cdot \log _{10}\left(\frac{(\text { Max value of Gray level })^{2}}{M S E}\right) \\
& M S E=\frac{1}{m n} \sum_{x=0}^{m-1} \sum_{y=0}^{n-1} \operatorname{steg} \_i m(x, y)-\operatorname{cov} e r_{-} i m(x, y) \text {. }
\end{aligned}
$$

$\mathrm{BER}=($ no. of wrong bit / no. of original bit $) * 100$

$N C=\sum_{x=0}^{m-1} \sum_{y=0}^{n-1} s t e g \_i m(x, y) * \operatorname{cov} e r \_i m(x, y) / \sum_{x=0}^{m-1} \sum_{y=0}^{n-1}\left(\left(\operatorname{cov} e r_{-} i m(x, y)\right)^{2}\right.$

فيما يأتي ندرج الجدول (1) والخاص بعملية التنفيذ والذي يعطي أفضلية واضحة للطريقة المقترحة على 
الجدول (1). نتائج تتفيذ الطريقة المقترحة على أنواع من الصور (BPG، PNG (BMP)

\begin{tabular}{|c|c|c|c|c|c|c|}
\hline BER & $\mathrm{NC}$ & PSNR & حجم الصورة & نوع الصورة & اسم الصورة & ت \\
\hline 0 & 1 & 66.6523 & $600 * 400$ & BMP & Bird & 1 \\
\hline 0.063 & 0.9785 & 59.8754 & $540 * 320$ & JPG & Boat & 2 \\
\hline 0 & 1 & 67.8621 & $800 * 600$ & BMP & Car & 3 \\
\hline 0.021 & 0.9854 & 59.9879 & $720 * 640$ & JPG & Ship & 4 \\
\hline 0.022 & 0.9821 & 59.8952 & $760 * 550$ & JPG & Butterfly & 5 \\
\hline 0 & 0.9993 & 64.7397 & $780 * 690$ & BMP & See & 6 \\
\hline 0.003 & 0.9991 & 63.8794 & $770 * 680$ & PNG & Eagle & 7 \\
\hline
\end{tabular}

تعد هذه الطريقة أفضل من (LSB) التقليدية للأسباب الآتية:

1- إذا قام المتطفل بتغير bit الأقل أهمية لكل المواقع في صورة الغطاء ففي طريقة LSB فانه لا يمكن

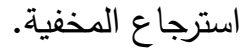

2- إذا تم تغيير بعض bit الأقل أهمية بسبب الضوضاء فباستخدام طريقتا في الاسترجاع فان الرسالة المخفية سوف تسترجع بصورة صحيحة وبدون أخطاء.

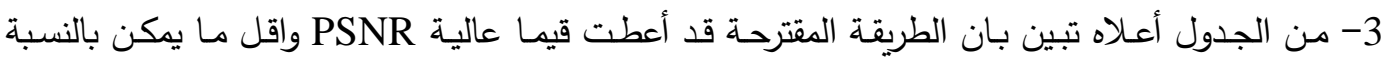
BER مما يعني الجودة العالية.

4- إن لغـة البرمجـة (Matlab) أعطت للعمل دعماً وجهداً مميزاً من ناحية الكفاءة والقدرة على التتفيذ

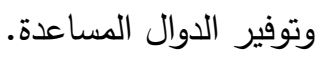
5- لموضوع الإخفاء أبعاد ومجالات عديدة ومتنوعة تفتح أفاقاً كثيرة للعمل فيه. 


\section{المصادر}

أحمد، فرح باسل و ندى جزيل ونجلاء طلب، (2009)، "حماية الملكية في الصسور"، بحث تخرج، كلية علوم الحاسوب والرياضيات، جامعة الموصل.

إسماعيل، رؤى سامي وعبد القادر، هبة هاني وعلي، شهلة عبد، (2009)، "'تطبيقات العلامة المائية في المجال المكاني"، بحث تخرج، كلية علوم الحاسوب والرياضيات، جامعة الموصل.

الحمامي، علاء حسين ومحمد علاء، (2008)، "إخفاء المعلومات"، إثراء للنشر والتوزيع.

حسن، زينة محمد ويونس، خليل احمد، (2009)، "الإخفاء في ملفات الانترنيت"، بحث تخرج، كلية علوم

الحاسوب والرياضيات، جامعة الموصل.

رفل أديب وسـالي عبد الجبار، (2010)، "إخفاء المعلومات باستخدام الثبكات العصبية الاصدطناعية"، بحث تخرج، كلية علوم الحاسوب والرياضيات، جامعة الموصل.

[7] RajKumar, Yadav, et al, (2011), "Anew Image Steganography Approach For Information Security Using Gray Level Images In Spatial Domain", International Journal On Computer Science And Engineering (IJCSE), July. 